\title{
Temperature Perturbation to Intrinsic Fluorescence of Phenyl Multimers in Glassy Polystyrene
}

\author{
Shing Wong \\ Polymer engineer, 32 York Street West, Ontario N0B 1S0. Email: swongph7@yahoo.ca \\ doi:10.5618/chem.2011.v2.n1.2 || Received: 20-1-2012, Accepted: 15-3-2012, Available online: 18-3-2012
}

\begin{abstract}
The newly discovered fluorescence in polystyrene is used as a probe to understand the phenyl motions in glassy polystyrene fine particles when temperature increases from room temperature to $62^{\circ} \mathrm{C}$. The large changes in fluorescence profiles (intensities and shapes) suggest profound phenyl motions and reorientations under $62^{\circ} \mathrm{C}$. When excite a single phenyl ring $\left(\lambda_{e x}=260 \mathrm{~nm}\right)$, proposed phenyl dimers $\left(\lambda_{\mathrm{ex}}=277 \mathrm{~nm}\right.$ and $296 \mathrm{~nm}$ ), and proposed tetramers $\left(\lambda_{\text {ex }}=360 \mathrm{~nm}\right.$ and $\left.380 \mathrm{~nm}\right)$, the heating has negative effects on the fluorescence intensities of all emissions, while the heating exhibits positive effects on the fluorescence emissions when excite proposed trimers $\left(\lambda_{\mathrm{ex}}=325 \mathrm{~nm}\right.$ and $\left.340 \mathrm{~nm}\right)$. This implies that the phenyl motions and reorientations under $62^{\circ} \mathrm{C}$ in the studied time frame are in the direction of favoring the formations of proposed phenyl trimers, at the cost of proposed phenyl dimers and tetramers. With continuous heating under $62{ }^{\circ} \mathrm{C}$, the emissions at $\lambda_{\text {ex }}$ $=277 \mathrm{~nm}$ showed different responses to the heat in its shorter wavelength region $(350-367 \mathrm{~nm})$ from its longer wavelength regions $(374-426 \mathrm{~nm})$, which is believed to be caused by the larger and increasing contributions of emissions from excited trimers $\left(\lambda_{\mathrm{ex}}=\mathbf{3 4 0} \mathrm{nm}\right)$ to the emissions at its longer wavelength side, through either indirect excitation of phenyl trimers by energy transfer or formation of excited trimers from some excited dimers, or both.
\end{abstract}

Keywords: polystyrene particles; phenyl multimers, phenyl motions; fluorescence; temperature; glassy state.

\section{Introduction}

As an important part of materials science and widely used in industrial, polymers display various macroscopic characteristics and features that are fundamentally controlled by the chain motions and dynamics due to the abundance of the internal degrees of freedom of polymer chains [1-6]. It is important to understand the molecular motions in a polymer chain for better material design. Especially, understanding the chain motions in a polymer in its glassy state is one of the unsolved mysteries of materials science and has attached much experimental and theoretical interest.

Recently new structured fluorescence emissions were reported in longer wavelengths $(360-460 \mathrm{~nm})$ than polystyrene excimer emission in polystyrene fine particles [7], cast film [7], and solutions [8]. The emissions and excitations were also assigned to different phenyl multimers based on the energy transfer diagram and previous studies [9]. In this work, the newlydiscovered fluorescence in polystyrene fine particles is used to probe molecular motions, particularly the phenyl motions, in its glassy state $\left(62^{\circ} \mathrm{C}\right)$.

\section{Materials and methods}

The molecular weights and polydispersities of polystyrene were $\mathrm{Mw}=223,000$ and $\mathrm{Mw} / \mathrm{Mn}=1.06$, respectively. Methanol and tetrahydrofuran (THF) in spectroscopic-grade were used without further purification. The polystyrene fine particles were prepared on a piece of quart plate following the method described in [7]. In order to minimize the effect of solvent evaporation on the fluorescence of the prepared polystyrene fine particles [7], the deposited polystyrene particles were allowed to dry in a vacuum intensively until the fluorescence intensity remains stable. The sample cell of the fluorescence spectrometer was preheated to $62{ }^{\circ} \mathrm{C}$, and then the polystyrene sample was put into the sample cell for measurements. The fluorescence of polystyrene particles was monitored over a $30 \mathrm{hrs}$ time period. The temperature of the sample cell was maintained at $62{ }^{\circ} \mathrm{C}$ through entire process of measurements.

Fluorescence spectra were recorded with a Jobin-Yvon SPEX F212 using the front-face mode at $62^{\circ}$. The slit widths were set to $1.0 \mathrm{~nm}$ for all measurements. To reduce noise, each reported spectrum was an average of two to three separate scans, moreover, some plotted spectra were reasonably smoothed.

\section{Results and discussion}

Fig. 1 presents the time dependence of fluorescence emissions of polystyrene fine particles at $62{ }^{\circ} \mathrm{C}$ at 
different excitation energies. The excitation energies correspond to the peaks shown in the excitation spectra in Fig. 2 of [7]: 277nm, 296nm, 325 nm, 340 nm, 360 $\mathrm{nm}, 380 \mathrm{~nm}$ and excitation energy of a single phenyl at $260 \mathrm{~nm}$. The time dependence of fluorescence emissions at $\lambda_{\mathrm{ex}}=260 \mathrm{~nm}$ is shown in both the left and the right columns for comparison. It should be noted that polystyrene particles required a certain time (estimated $0.5 \mathrm{hr}$ ) to reach the destination temperature $62^{\circ} \mathrm{C}$ when the sample stored in room temperature was put in $62{ }^{\circ} \mathrm{C}$ sample cell, and the polystyrene particles before reaching $62{ }^{\circ} \mathrm{C}$ were in different temperatures between room temperature $\left(\sim 25^{\circ} \mathrm{C}\right)$ and $62^{\circ} \mathrm{C}$. The polystyrene particles measured under zero heating time should be considered to be at room temperature $25^{\circ} \mathrm{C}$.

At $\lambda_{\mathrm{ex}}=260,277$ and $296 \mathrm{~nm}$, the emission profiles show the strongest peaks at $367 \mathrm{~nm}$ at room temperature ( $0 \mathrm{hr}$ heating), but their relative intensities of the major emissions changed differently with heating. At $\lambda_{\mathrm{ex}}=260$ $\mathrm{nm}$, all the strong emissions between 350-385 $\mathrm{nm}$ decrease, and the intensities of the peaks located at 367 $\mathrm{nm}, 374 \mathrm{~nm}$ and $385 \mathrm{~nm}$ became very similar after 30 hrs' heating at $62^{\circ} \mathrm{C}$. The emission peaks located at 374 $\mathrm{nm}$ and $385 \mathrm{~nm}$ became dominant in the case of $\lambda_{\text {ex }}$ $=277 \mathrm{~nm}$, while the strongest peak remains at $367 \mathrm{~nm}$ at $\lambda_{\mathrm{ex}}=296 \mathrm{~nm}$. Different from the cases at $\lambda_{\mathrm{ex}}=260,277$ and $296 \mathrm{~nm}$, the emissions at $\lambda_{\mathrm{ex}}=325$ and $340 \mathrm{~nm}$ exhibited large intensity increase in the first two hrs of heating. After 2 hrs's heating, the emissions showed much slow increase and then a slight decrease in their intensities. Similar to the cases at $\lambda_{\mathrm{ex}}=260,277$ and 296 $\mathrm{nm}$, the fluorescence intensities of emissions at $\lambda_{\text {ex }}=360$ and $380 \mathrm{~nm}$ decrease with heating. However the decrease was only very obvious in the first two hours of heating, which is different from the cases at $\lambda_{\mathrm{ex}}=260$, 277 and $296 \mathrm{~nm}$.

When excite a sing phenyl group at $\lambda_{\mathrm{ex}}=260 \mathrm{~nm}$, there are two possible paths leading to the emissions at longer wavelength than the excimer emission at $325 \mathrm{~nm}$ : 1) the energy transfer from the emissions of single phenyl group and excimers; 2) forming the excited multimers by interacting of an excited single phenyl with surrounding phenyl groups. In either path, it requires large density of phenyl aggregations (aggregations of phenyl groups in proper geometry and distance) to produce strong emissions. The decrease in fluorescence at $\lambda_{\mathrm{ex}}=260 \mathrm{~nm}$ with heating suggests that the temperature increasing from $25{ }^{\circ} \mathrm{C}$ to $62{ }^{\circ} \mathrm{C}$ can reduce the density of these phenyl aggregations with emitted fluorescence at longer wavelengths (either directly or indirectly). Polystyrene is in a glassy state at both $25{ }^{\circ} \mathrm{C}$ and $62{ }^{\circ} \mathrm{C}$, and molecular motions in the glassy sate are largely restricted to vibrations and short- range rotational motions. It was reported that the most prevalent motion in glassy polystyrene is restricted phenyl rotation with a sizable average jump angle [10]. The high-frequency ring flips in combination with high frequency main-chain rotational reorientation occur at a small fraction of sites within some of the polystyrenes [10]. Based on this previous study, I propose that the thermal energy gained from temperature increase can promote these phenyl rotations and reorientation to relieve local steric constraints imposed by the hydrophobic interactions during polystyrene reprecipitation and fast solvent removal. These motions can either conquer the molecular interactions that hold the fluorous phenyl mutlimers together to dissociate multimers into free phenyl rings, or facilitate the formations of only one or two preferred mutlimers at the cost of other mutlimers. In both cases, some energy transfer paths are cut, which results in weaker emissions. The same observations are found for the fluorescence emissions at excitation energies of $\lambda_{\mathrm{ex}}=(277 \mathrm{~nm}$ and $296 \mathrm{~nm})$, and $\lambda_{\text {ex }}=(360 \mathrm{~nm}$ and $380 \mathrm{~nm})$ which correspond to the excitations of proposed phenyl dimers and tetramers, respectively [9].

However at $\lambda_{\mathrm{ex}}=325 \mathrm{~nm}$ and $340 \mathrm{~nm}$, two excitation energies corresponding to proposed excitations of phenyl trimers [9], the intensities of fluorescence emissions increase greatly in the first 2 hours' heating. This suggests that the increased thermal energy can facilitate the formations of the proposed phenyl trimers. This is an interesting phenomenon. It is not sure whether these new phenyl trimers are formed from the existed phenyl dimers or tetramers, or three free phenyl groups, but it seems that the phenyl trimers are more thermal stable than the phenyl dimers and tetramers within the studied time frame and temperature.

With increasing heating time, the emissions at $\lambda_{\text {ex }}$ $=277 \mathrm{~nm}$ shows much less decrease in intensity in the range of $374-394 \mathrm{~nm}$ than the range of $350-367 \mathrm{~nm}$, but this is not observed for the emissions at $\lambda_{\mathrm{ex}}=260$ and $296 \mathrm{~nm}$. Considering the positive effects of heating on the emissions (374-394 $\mathrm{nm}$ ) at $\lambda_{\mathrm{ex}}=325 \mathrm{~nm}$ and $340 \mathrm{~nm}$, this might indicate that energy transfer path(s) involving the indirect excitation of phenyl trimers contributes much more to the overall energy transfers occurred at $\lambda_{\text {ex }}=277 \mathrm{~nm}$ than at $\lambda_{\text {ex }}=260 \mathrm{~nm}$ and $296 \mathrm{~nm}$. The heating can facilitate the formation of phenyl trimers and increase the emissions in the energy range of 374$394 \mathrm{~nm}$, which can compensate for the intensity loss in the emissions of $374-394 \mathrm{~nm}$ at $\lambda_{\text {ex }}=277 \mathrm{~nm}$ from other emission paths.

The intensity variations of major fluorescence emissions as pointed by arrows in Fig.1 (f 325, f 350 and so on) with heating time at $62{ }^{\circ} \mathrm{C}$ are plotted in Fig. 2. 

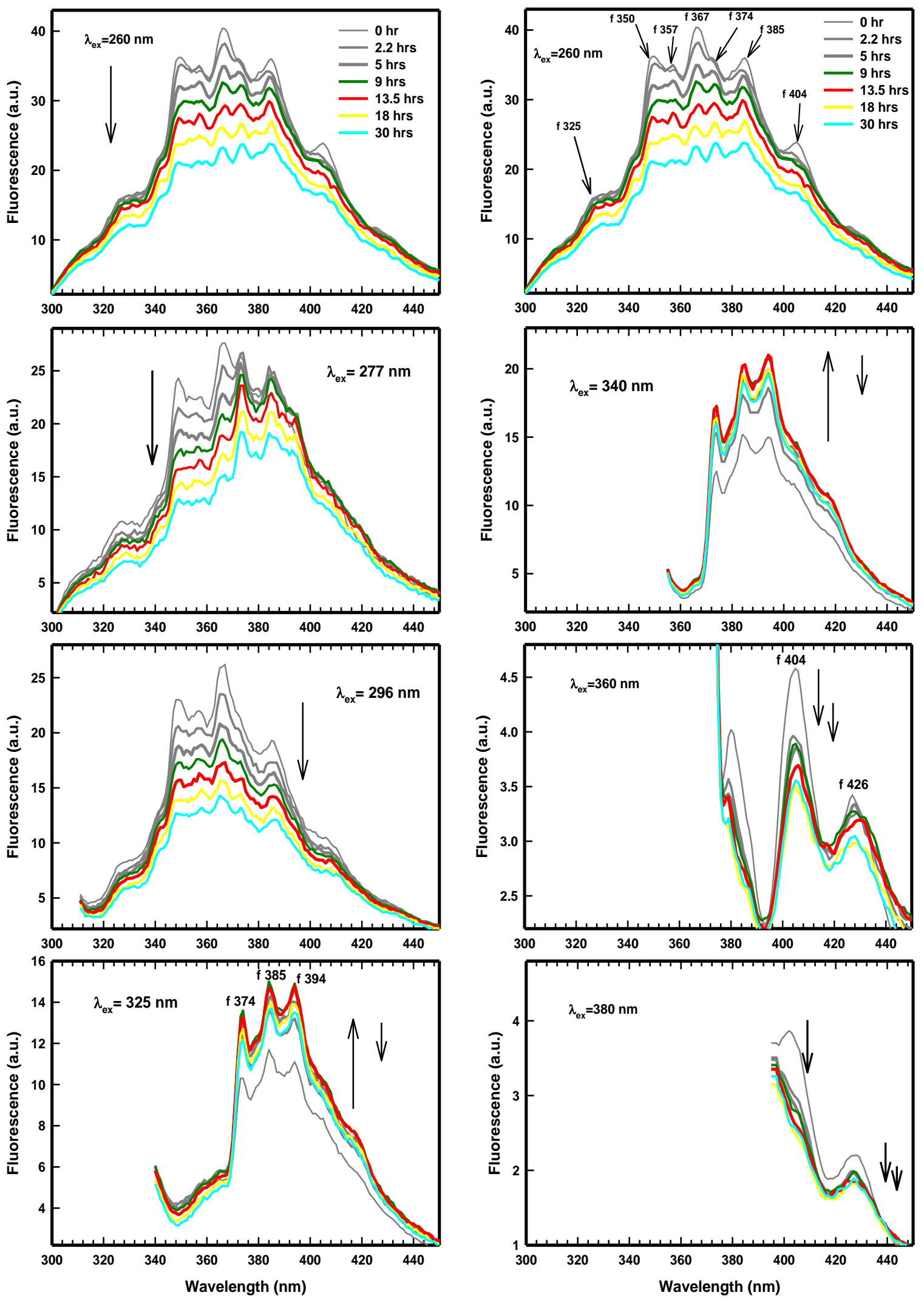

Fig. 1. The time dependence of fluorescence at different excitation energies with heating at $62^{\circ} \mathrm{C}$. The Fluorescence intensities are divided by the same number. 

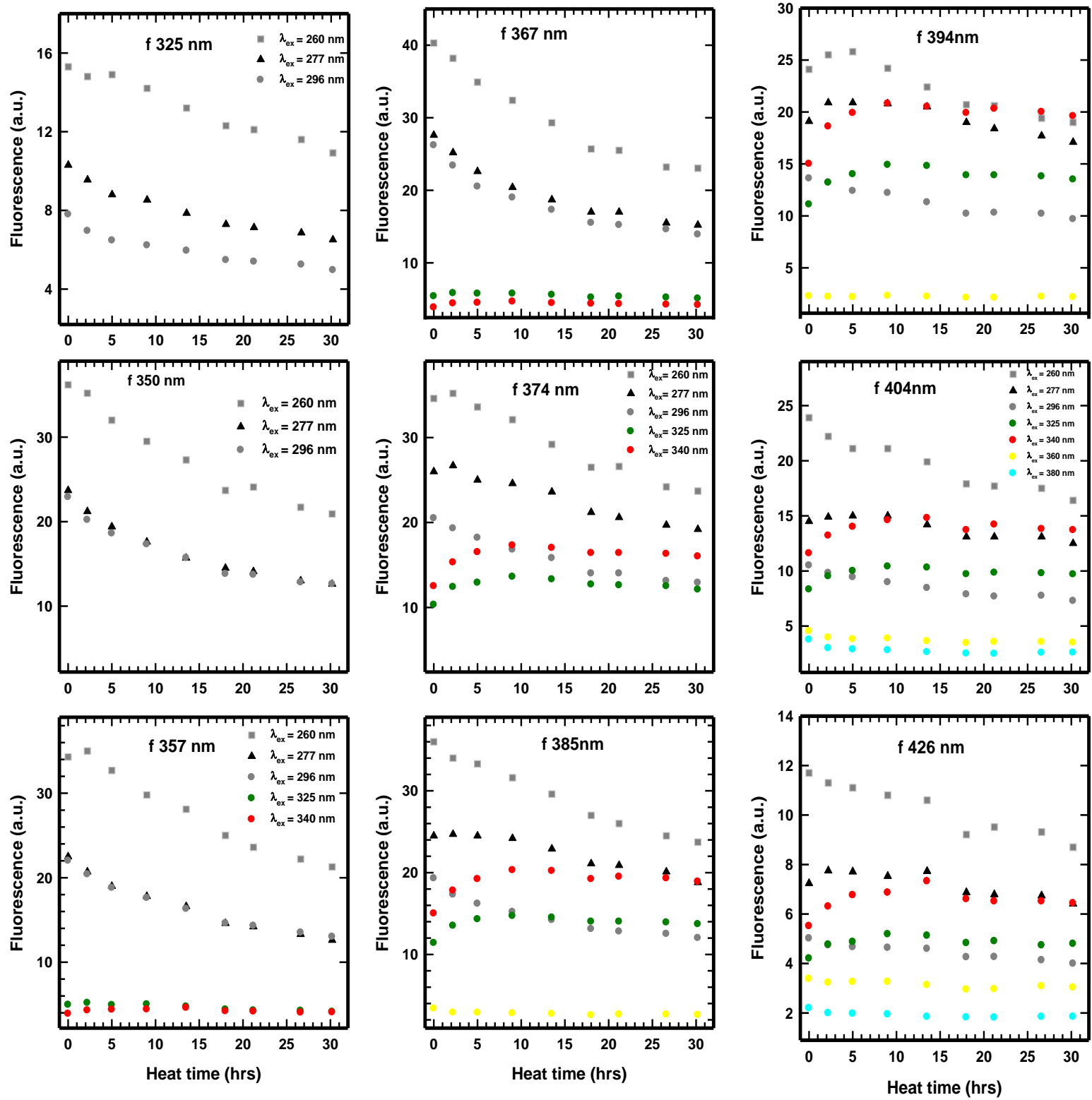

Fig. 2. The variations of fluorescence at different excitation energies over heating time at $62^{\circ} \mathrm{C}$. Different symbols are used for fluorescence at different excitation energies (see the legend of plot $\mathbf{f} \mathbf{4 0 4} \mathbf{~ n m}$ for a complete list of symbols). f $325 \mathrm{~nm}$ means the fluorescence emission at $325 \mathrm{~nm}$, and so on. These fluorescence are indicated by arrows in Fig. 1. The Fluorescence intensities are divided by the same number.

plot, different symbols correspond to the same fluorescence at different excitation energies (see the legend of the plot of Fluorescence at $404 \mathrm{~nm}$.). One should keep in mind that most of these emissions are overlapped and hard to deconvolute, thus the intensity of one emission peak has the contributions from others, especially the emissions with closer wavelengths. Although the intensities of these emissions are only approximations, their change over time can still provide some insight into the fluorescence changes due to phenyl motions in polystyrene.

All the fluorescence emissions show strongest intensity with the excitation energy at $260 \mathrm{~nm}\left(\lambda_{\mathrm{ex}}=260 \mathrm{~nm}\right.$, grey squares). This suggests that the excitation of the single phenyl groups in polystyrene particles leads to the most efficient and strongest fluorescence, regardless of which multimers the emissions emit from.

At excitation energies $\lambda_{\mathrm{ex}}=260,277$ and $296 \mathrm{~nm}$, the excimer emissions ( f $325 \mathrm{~nm}$ ) shows different inten- 
sities and their intensities decrease with increasing heating time. The rates of decrease become slightly flatter with increasing excitation energies. The fluorescence at $350 \mathrm{~nm}$ (f 350) and $357 \mathrm{~nm}$ (f 357) also shows similar intensity decreases as $\mathrm{f} 325$, but different from f $325 \mathrm{~nm}$, both f 350 and f 357 have almost the same intensities at the two different excitation energies $277 \mathrm{~nm}$ and $296 \mathrm{~nm}$ during the course of heating, as indicated by the overlapping of triangles $\left(\lambda_{\mathrm{ex}}=277 \mathrm{~nm}\right)$ and grey circles $\left(\lambda_{\mathrm{ex}}=296 \mathrm{~nm}\right)$ in the figures of $\mathrm{f} 350$ and $f$ 357. This suggests that under the excitation energies of $277 \mathrm{~nm}$ and $296 \mathrm{~nm}$, both f 350 and f 357 are emitted from the same excited dimers and from the same energy transfer paths, if there are any. However, for the emissions with longer wavelengths, they show increasing differences in their heating responses at two excitation energies at 277 and $296 \mathrm{~nm}$. The f 367 shows slight intensity differences at $\lambda_{\mathrm{ex}}=277$ and $296 \mathrm{~nm}$, but all the other fluorescence (f 374 - f 426) shows much bigger differences in their heating responds at $\lambda_{\mathrm{ex}}=277$ and $296 \mathrm{~nm}$. Not only are their intensities quiet different at $\lambda_{\mathrm{ex}}=277$ and $296 \mathrm{~nm}$, but also their changes over heating time have different trends. The f 374 - f 426 all decrease with heating time at $\lambda_{\mathrm{ex}}=296 \mathrm{~nm}$, but they tend to increase slightly first and then decrease at $\lambda_{\mathrm{ex}}=$ $277 \mathrm{~nm}$. This suggests the excitation of $277 \mathrm{~nm}$ and 296 $\mathrm{nm}$ have different contributions to the emissions at longer wavelengths $374 \mathrm{~nm}-426 \mathrm{~nm}$ during the course of heating, but the same contributions to the emissions at $350 \mathrm{~nm}$ and $357 \mathrm{~nm}$, and very similar contributions to the emission at $367 \mathrm{~nm}$.

The heating have negative effects on the fluorescence intensities of most emissions, and the only positive effects are found on the emissions resulting from the excitation at $325 \mathrm{~nm}$ and $340 \mathrm{~nm}$, as indicated by the curves in green circles $(325 \mathrm{~nm})$ and red circles (340 $\mathrm{nm}$ ), respectively, in Fig. 2. This positive effect is most profound on the emissions at $384 \mathrm{~nm}$ and $394 \mathrm{~nm}$, especially the emission at $394 \mathrm{~nm}$ which the fluorescence intensities are raised to the same level (the highest level) as the excitation at $260 \mathrm{~nm}$ after a certain heating time. It is interesting to observe that the heating can raise the fluorescence intensities of each emission of f 385 , f 404 and f 426 at $\lambda_{\text {ex }}=340$ to the same intensity as those at $\lambda_{\mathrm{ex}}=277$, as indicated by the overlapping of triangles $\left(\lambda_{\mathrm{ex}}=277 \mathrm{~nm}\right)$ and red circles $\left(\lambda_{\mathrm{ex}}=340 \mathrm{~nm}\right)$ appeared after a period of relative longer heating time. This suggests that the excitation at $277 \mathrm{~nm}$ can either facilitate the formation of excited trimers. This confirms the above conclusion that with heating, the emissions from excited trimers has bigger or complete contributions to the emissions in longer wavelengths at the excitation of $277 \mathrm{~nm}$ (excited dimers), through either indirect excitation of phenyl trimers by energy transfer or formation of excited trimers from some excited dimers, or both.

\section{Summary}

To probe the phenyl motions in glassy polystyrene, the polystyrene fine particles after intensive vacuum drying to remove solvent were investigated regarding their fluorescent responses to heating at $62{ }^{\circ} \mathrm{C}$. In glassy polystyrene fine particles under either room temperature or $62{ }^{\circ} \mathrm{C}$, it was found that the excitation of the single phenyl groups $\left(\lambda_{\mathrm{ex}}=260 \mathrm{~nm}\right)$ in polystyrene particles leads to the most efficient and strongest fluorescence, regardless of which multimers the emissions emit from. Compared with the fluorescence emissions at room temperature $\left(\sim 25^{\circ} \mathrm{C}\right)$, most emissions showed decreased intensities with heating at $62{ }^{\circ} \mathrm{C}$, with the exception of the emissions from the excitations at $325 \mathrm{~nm}$ and 340 $\mathrm{nm}$ (proposed phenyl trimers [9]). This implies that there are profound phenyl motions and reorientations occurred in polystyrene fine particles when heat under $62{ }^{\circ} \mathrm{C}$. It can be also concluded that the thermal energy gain from temperature increase can facilitate the formations of proposed phenyl trimers by phenyl motions and reorientations in the studied time period, at the cost of proposed phenyl dimers and tetramers. With continuous heating under $62{ }^{\circ} \mathrm{C}$, the emissions at $\lambda_{\mathrm{ex}}=$ $277 \mathrm{~nm}$ showed different responses to the heat in shorter wavelengths (f 350- f 367) than at longer wavelengths (f 374- $\mathrm{f} \mathrm{426),} \mathrm{which} \mathrm{is} \mathrm{believed} \mathrm{to} \mathrm{be} \mathrm{caused} \mathrm{by} \mathrm{the} \mathrm{larger}$ and increasing contributions to the emission at longer wavelengths from excited trimers, through either indirect excitation of phenyl trimers by energy transfer or formation of excited trimers from some excited dimers, or both. This can also explain the observation that after a period of relative longer heating time, when excite at $\lambda_{\mathrm{ex}}=340 \mathrm{~nm}$, the fluorescence intensities of each emission at longer wavelengths among 385nm, 404 $\mathrm{nm}$ and $426 \mathrm{~nm}$ were all increased to the same level as the emissions at $\lambda_{\text {ex }}=277$. In conclusion, this work demonstrates that we can use the rich intrinsic fluorescence in polystyrene as a probe for its chain motions without the extrinsic fluorescence labels.

The fluorescence fluctuations with heating at $62{ }^{\circ} \mathrm{C}$ is only reported for the $30 \mathrm{hrs}$ ' heating period here, although the fluorescence was monitored in longer period. It was found that fluorescence intensities ultimately decreased for all the emissions at all the selected excitation energies when the polystyrene particles were heated long enough. At the same time, the baselines of the fluorescence spectra were also shifted down. This suggests that there might be some other contributes rather than molecule motions attributed for such changes occurred in long heating time. 


\section{References}

[1] Flory, P. J. Staristical Afechanics of Chain Molecules, 1969, Interscience: New York.

[2] Yamakawa, H. Modern Theory of Polymer Solutions, 1971, Harper \& Rov,l; New York.

[3] de Gennes, P. -G. Scaling Concepts in Polymer Physics, 1979, Cornell Univ. Press: Ithaca, N.Y..

[4] Ferry, J. D. Viscoelastic Properties of Polymers 3rd Ed., 1980, lohn Wiley and Sons:New York.

[5] Doi, M.; Edwards, S. F. The Theory of Polymer Dynamics, 1986, Clarendon Press:Oxford.
[6] Matsuoka, S. Relaxation Phenomena in Polymers, 1992, Carl Hanser Verlag: New York.

[7] Kuo, A. CheM, 2011, 1, 1-8.

http://dx.doi.org/10.5618/chem.2011.v1.n1.1

[8] Kuo, A. CheM, 2011, 1, 80-86.

http://dx.doi.ora/10.5618/chem.2011.v1.n1.9

[9] Kuo, A. CheM, 2011, 1, 40-51.

http://dx.doi.org/10.5618/chem.2011.v1.n1.6

[10]Schaefer, J.; Sefcik, M. D.; Stejskal, E. O.; McKay, R. A.; Thomas Dixont, W.; Cais, R. E. Macromolecules, 1984, 17, 1107-1118.

http://dx.doi.org/10.1021/ma00136a001 